\title{
A terminal molybdenum carbide prepared by methylidyne deprotonation
}

\author{
Jonas C. Peters, Aaron L. Odom and Christopher C. Cummins $* \dagger$ \\ Department of Chemistry, Massachusetts Institute of Technology, 77 Massachusetts Avenue, Cambridge, MA 02139, USA
}

The carbide anion $\left[\mathrm{CMo}\{\mathrm{N}(\mathrm{R}) \mathrm{Ar}\}_{3}\right]^{-}\left[\mathrm{R}=\mathrm{C}\left(\mathrm{CD}_{3}\right)_{2} \mathrm{CH}_{3}, \mathrm{Ar}\right.$ $\left.=\mathrm{C}_{6} \mathrm{H}_{3} \mathrm{Me}_{2}-3,5\right]$, is obtained by deprotonation of the corresponding methylidyne compound, [HCMo $\left.\{\mathrm{N}(\mathrm{R}) \mathrm{Ar}\}_{3}\right]$, and is characterized by $\mathrm{X}$-ray diffraction as its $\{\mathrm{K}($ benzo15-crown-5) $\}_{2}{ }^{+}$salt, thereby providing precedent for the carbon atom as a terminal substituent in transition-metal chemistry.

Recent work has shown that the molybdenum-nitrogen multiple bond in the nitride species $\left[\mathrm{NMo}\{\mathrm{N}(\mathrm{R}) \mathrm{Ar}\}_{3}\right]$ $\left[\mathrm{R}=\mathrm{C}\left(\mathrm{CD}_{3}\right)_{2} \mathrm{CH}_{3}, \mathrm{Ar}=\mathrm{C}_{6} \mathrm{H}_{3} \mathrm{Me}_{2}-3,5\right]$ is exceedingly strong, and that this complex is quite robust. ${ }^{1,2}$ Recognizing that the anionic carbide $\left[\mathrm{CMo}\{\mathrm{N}(\mathrm{R}) \mathrm{Ar}\}_{3}\right]^{-}$would be isoelectronic with $\left[\mathrm{NMo}\{\mathrm{N}(\mathrm{R}) \mathrm{Ar}\}_{3}\right]$, we set out to synthesize it. As described herein, a successful synthesis was accomplished via deprotonation of the $\mathrm{d}^{0}$ methylidyne complex $\left[\mathrm{HCMo}\{\mathrm{N}(\mathrm{R}) \mathrm{Ar}\}_{3}\right]$. Previously reported molecular carbide complexes feature carbon atoms with at least two nearest neighbors. ${ }^{3-10}$

Three-coordinate $\left[\mathrm{Mo}\{\mathrm{N}(\mathrm{R}) \mathrm{Ar}\}_{3}\right] \mathbf{1}$ was treated with $99 \%$ ${ }^{13} \mathrm{C}$ carbon monoxide to give the paramagnetic $\left(\mu_{\text {eff }}=1.73 \mu_{\mathrm{B}}\right)$ carbonyl complex $\left[\left(\mathrm{O}^{13} \mathrm{C}\right) \mathrm{Mo}\{\mathrm{N}(\mathrm{R}) \mathrm{Ar}\}_{3}\right] 2\left(v_{\mathrm{CO}} 1797 \mathrm{~cm}^{-1}\right)$ as a brown solid in $88 \%$ yield. $\$ \$$ One-electron reduction of 2 was effected with sodium amalgam in tetrahydrofuran (thf) giving $\left[\left(\mathrm{NaO}^{13} \mathrm{C}\right) \mathrm{Mo}\{\mathrm{N}(\mathrm{R}) \mathrm{Ar}\}_{3}\right]_{2} 3\left[\delta\left({ }^{13} \mathrm{C}\right) 243.0\right]$ in essentially quantitative yield. $\mathbf{3}$ was obtained either as a solvent-free dimer or as an ether or thf solvate. In situ generated $\mathbf{3}$ was found to be sufficiently pure for synthetic purposes. Reaction of $\mathbf{3}$ with pivaloyl chloride ${ }^{11}$ provided the 'carbido pivalate' complex $\left[\mathrm{Bu}{ }^{\mathrm{t}} \mathrm{C}(\mathrm{O}) \mathrm{O}\left({ }^{13} \mathrm{C}\right) \mathrm{Mo}\{\mathrm{N}(\mathrm{R}) \mathrm{Ar}\}_{3}\right] 4\left[\delta\left({ }^{13} \mathrm{C}\right) 217.2\right]$ in $82 \%$ yield as a beige crystalline solid.I[ Removal of the pivalate moiety in 4 was effected by treatment with sodium in thf giving, according to ${ }^{13} \mathrm{C}$ NMR analysis, a mixture of 'sodium carbide' $\left[\mathrm{Na}\left({ }^{13} \mathrm{C}-\right.\right.$ ) $\left.\mathrm{Mo}\{\mathrm{N}(\mathrm{R}) \mathrm{Ar}\}_{3}\right]_{2} 5\left[\delta\left({ }^{13} \mathrm{C}\right) 474.2\right]$, methylidyne $\left[\mathrm{H}\left({ }^{13} \mathrm{C}\right) \mathrm{Mo}\{\mathrm{N}-\right.$ (R) $\left.\mathrm{Ar}_{3}\right] \mathbf{6}\left[\delta\left({ }^{13} \mathrm{C}\right) 287.5,{ }^{1} J_{\mathrm{CH}} 157 \mathrm{~Hz}\right]$ and 3 . Treatment of the mixture with an excess of acetonitrile converted $\mathbf{5}$ to $\mathbf{6}$, solubilized $\mathbf{3}$ and precipitated $\mathbf{6}$ in essentially pure form. Methylidyne 6, an off-white solid, was isolated by this procedure in 53\% yield from 2.|| Several other routes to the terminal methylidyne functionality have been documented. ${ }^{12-17}$

Deprotonation of $\mathbf{6}$ was effected by benzylpotassium in thf, 18 giving the potassium-molybdenum carbido complex $\left[\mathrm{K}\left({ }^{13} \mathrm{C}\right) \mathrm{Mo}\{\mathrm{N}(\mathrm{R}) \mathrm{Ar}\}_{3}\right]_{2} 7\left[\delta\left({ }^{13} \mathrm{C}\right) 494.5\right]^{19,20}$ in $69 \%$ yield after recrystallization from pentane-thf.** In thf solution 7 presumably exists as a thf solvate, but the indicated thf-free, benzene-soluble dimer stabilized by intramolecular $\mathrm{K}^{+}$-arene interactions $^{21}$ is readily obtained. Dimer 7 has been shown by $\mathrm{X}$-ray crystallography to possess a central $\mathrm{K}_{2}$ (carbide) ${ }_{2}$ square array situated about a crystallograpic inversion center. ${ }^{22,23}$

Treatment in thf of the potassium-molybdenum carbide 7 with 2,2,2-crypt ${ }^{24}$ led to formation of the thf-soluble, benzeneinsoluble salt $[\mathrm{K}(2,2,2$-crypt $)]\left[{ }^{13} \mathrm{CMo}\{\mathrm{N}(\mathrm{R}) \mathrm{Ar}\}_{3}\right] \mathbf{8}\left[\delta\left({ }^{13} \mathrm{C}\right)\right.$ 482.8]. $\dagger \dagger$ The ${ }^{1} \mathrm{H}$ NMR spectrum of $\mathbf{8}$ in $\left[{ }^{2} \mathrm{H}_{8}\right]$ thf showed the expected four sharp resonances for the threefold-symmetric anion, along with the three signals characteristic of the $[\mathrm{K}(2,2,2 \text {-crypt })]^{+}$cation. The curious fact that the ${ }^{13} \mathrm{C}$ NMR signal for the carbido carbon in $\mathbf{8}$ is broad ( $\left[{ }^{2} \mathrm{H}_{8}\right]$ thf, $\Delta v_{1 / 2} 1400$ $\mathrm{Hz}$ ) contrasts with the situation for 7, which (in $\mathrm{C}_{6} \mathrm{D}_{6}$ ) exhibits a sharp signal.

A salt of the $\left[\mathrm{CMo}\{\mathrm{N}(\mathrm{R}) \mathrm{Ar}\}_{3}\right]^{-}$anion amenable to X-ray crystallography, 9, was obtained upon treatment of $\mathbf{7}$ with 2 equiv. (per K) of benzo-15-crown-5. ${ }^{25}$ Crystals of salt 9, which exhibited solubility characteristics similar to $\mathbf{8}$, were obtained from thf-pentane. $\neq$ The X-ray diffraction study of 9 revealed discrete and separate $\left[\mathrm{CMo}\{\mathrm{N}(\mathrm{R}) \mathrm{Ar}\}_{3}\right]^{-}$anions and $[\mathrm{K}($ benzo15-crown-5) $\left.{ }_{2}\right]^{+}$cations (Fig. 1).\$§ The molybdenum-carbon distance in 9 was found to be 1.713(9) $\AA$, at the low end of the known range for Mo-C multiple bonds. ${ }^{26}$ The three N(R)Ar
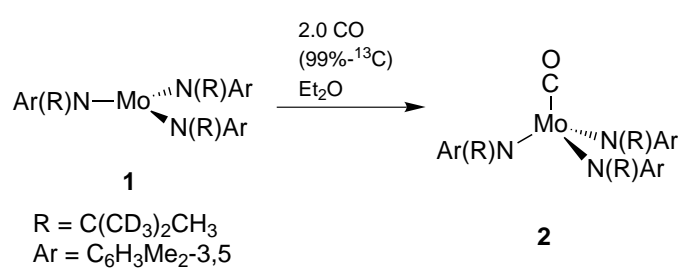
ii $1.0 \mathrm{Bu}^{\mathrm{t}} \mathrm{C}(\mathrm{O}) \mathrm{Cl}$ $\mathrm{Ar}=\mathrm{C}_{6} \mathrm{H}_{3} \mathrm{Me}_{2}-3,5$
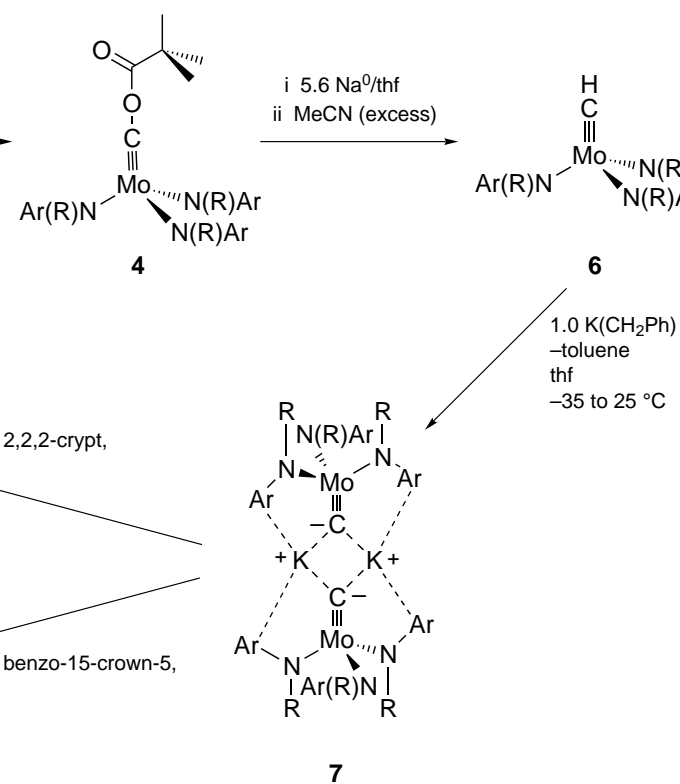

Scheme 1 

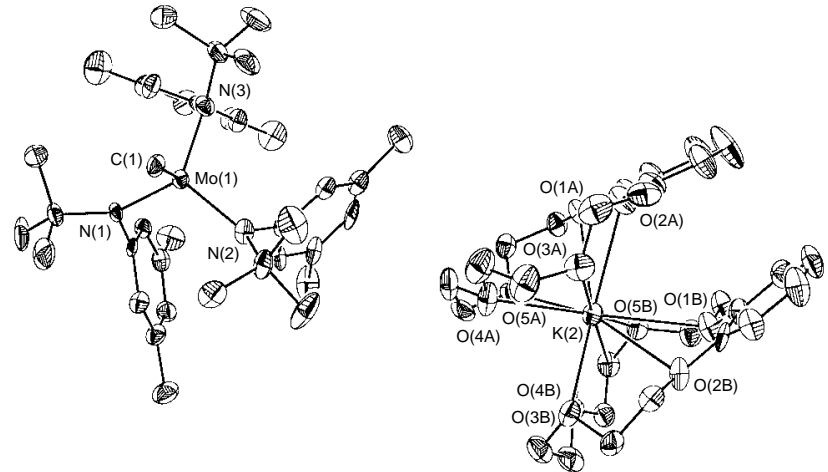

Fig. 1 Structural representation of $\left[\mathrm{K}(\text { benzo-15-crown-5 })_{2}\right][\mathrm{CMo}\{\mathrm{N}$ $\left.(\mathrm{R}) \mathrm{Ar}_{3}\right] \mathbf{9}$ with thermal ellipsoids at the $35 \%$ probability level. Selected bond lengths $(\AA)$ and angles $\left({ }^{\circ}\right)$ : $\operatorname{Mo}(1)-\mathrm{C}(1)$ 1.713(9), $\operatorname{Mo}(1)-\mathrm{N}(1)$ $2.008(6), \operatorname{Mo}(1)-\mathrm{N}(2) 2.010(7), \operatorname{Mo}(1)-\mathrm{N}(3) 2.013(6) ; \mathrm{C}(1)-\mathrm{Mo}(1)-\mathrm{N}(1)$ $102.7(3), \quad \mathrm{C}(1)-\mathrm{Mo}(1)-\mathrm{N}(2) \quad 103.9(3), \quad \mathrm{N}(1)-\mathrm{Mo}(1)-\mathrm{N}(2) \quad 114.7(3)$ C(1)-Mo(1)-N(3) 103.8(3), N(1)-Mo(1)-N(3) 116.9(3), N(2)-Mo(1)$\mathrm{N}(3) 112.6(3)$

ligands in $\left[\mathrm{CMo}\{\mathrm{N}(\mathrm{R}) \mathrm{Ar}\}_{3}\right]^{-}$adopt a propeller motif with the aryl rings $\pi$ stacked $^{27}$ on one side of the molecule and the tertbutyl groups on the other side forming a protective 'pocket' about the Mo-C multiple bond. The gross structural and conformational features of $\left[\mathrm{CMo}\{\mathrm{N}(\mathrm{R}) \mathrm{Ar}\}_{3}\right]^{-}$were anticipated based on structures of the related nitrido and phosphido derivatives $\left.\left[\mathrm{NMo}\left\{\mathrm{N}(\mathrm{Bu})^{\mathrm{t}}\right) \mathrm{Ph}\right\}_{3}\right]^{1}$ and $\left[\mathrm{PMo}\{\mathrm{N}(\mathrm{R}) \mathrm{Ar}\}_{3}\right] .^{.28}$

For funding this work we thank the Packard foundation, the Alfred P. Sloan Foundation, the National Science Foundation (CAREER Award CHE=9501992), 3M, DuPont, and Union Carbide. J. C. P. is grateful for a Department of Defense Graduate Research Fellowship. This work is dedicated to the memory of Professor Jeremy K. Burdett.

\section{Footnotes and References}

*E-mail: ccummins@mit.edu

$\dagger$ Alfred P. Sloan Fellow, 1997-2000.

\$ All manipulations were carried out under an atmosphere of dry nitrogen using solvents purified by standard methods.

$\S$ Other data for $2:{ }^{2} \mathrm{H}$ NMR $\left(46 \mathrm{MHz}\right.$, pentane, $\left.25{ }^{\circ} \mathrm{C}\right) ; \delta 8.0\left[\mathrm{~s}, \Delta v_{1 / 2} 14\right.$ $\left.\mathrm{Hz}, \mathrm{C}\left(\mathrm{CD}_{3}\right)_{2}\right] ; \mu_{\mathrm{eff}}\left(300 \mathrm{MHz}, 25^{\circ} \mathrm{C}, \mathrm{C}_{6} \mathrm{D}_{6}\right)=2.2 \mu_{\mathrm{B}}$. FTIR (heptane, $\mathrm{KBr}$ ); $v\left({ }^{13} \mathrm{CO}\right) 1797 \mathrm{~cm}^{-1}$. Anal. Calc. for $\mathrm{C}_{37} \mathrm{H}_{36} \mathrm{D}_{18} \mathrm{MoN}_{3} \mathrm{O}$ : C, 66.24; H, 8.11; N, 6.26. Found: C, 65.85; H, 8.44; N, 6.19\%.

II The reaction to produce 4 was carried out using $6.5 \mathrm{mmol}$ of 2 . Other data for 4: ${ }^{1} \mathrm{H}$ NMR $\left(300 \mathrm{MHz}, \mathrm{C}_{6} \mathrm{D}_{6}, 25^{\circ} \mathrm{C}\right) ; \delta 6.67\left(\mathrm{~s}, 3 \mathrm{H}, \mathrm{C}_{6} \mathrm{H}_{3} \mathrm{Me}_{2}, p-\mathrm{H}\right)$, $6.09\left(\mathrm{~s}, 6 \mathrm{H}, \mathrm{C}_{6} \mathrm{H}_{3} \mathrm{Me}_{2} o-\mathrm{H}\right), 2.10\left(\mathrm{~s}, 18 \mathrm{H}, \mathrm{C}_{6} \mathrm{H}_{3} M e_{2}\right), 1.50[\mathrm{~s}, 9 \mathrm{H}$, $\mathrm{C}\left(\mathrm{CD}_{3}\right)_{2} \mathrm{CH}_{3}$ ], 1.22 [s, $9 \mathrm{H}, \mathrm{C}\left(\mathrm{CH}_{3}\right)_{3}$ ]. Anal. Calc. for $\mathrm{C}_{42} \mathrm{H}_{45} \mathrm{D}_{18} \mathrm{MoN}_{3} \mathrm{O}_{2}$ : $\mathrm{C}, 66.73 ; \mathrm{H}, 8.40 ; \mathrm{N}, 5.56$. Found: C, 66.64; H, 8.24; N, 5.79\%. $\|$ The reaction to produce 6 was carried out using $2.66 \mathrm{mmol}$ of 4 . Other data for 6: ${ }^{1} \mathrm{H}$ NMR $\left(300 \mathrm{MHz}, \mathrm{C}_{6} \mathrm{D}_{6}, 25{ }^{\circ} \mathrm{C}\right) ; \delta 6.64\left(\mathrm{~s}, 3 \mathrm{H}, \mathrm{C}_{6} \mathrm{H}_{3} \mathrm{Me}_{2}, p-\mathrm{H}\right)$, $6.00\left(\mathrm{~s}, 6 \mathrm{H}, \mathrm{C}_{6} \mathrm{H}_{3} \mathrm{Me}_{2}, o-\mathrm{H}\right), 5.66\left[\mathrm{~d}, 1 \mathrm{H}, \mathrm{Mo}(\mathrm{CH}),{ }^{1} J_{\mathrm{CH}} 157 \mathrm{~Hz}\right], 2.07$ (s, $\left.18 \mathrm{H}, \mathrm{C}_{6} \mathrm{H}_{3} M e_{2}\right), 1.49$ [s, $\left.9 \mathrm{H}, \mathrm{C}\left(\mathrm{CD}_{3}\right)_{2} \mathrm{CH}_{3}\right]$. Anal. Calc. for $\mathrm{C}_{37} \mathrm{H}_{37} \mathrm{D}_{18} \mathrm{MoN}_{3}$ : C, 67.75; H, 8.45; N, 6.41. Found: C, 67.61; H, 8.29; N, $6.72 \%$.

** The reaction to produce 7 was carried out using $1.164 \mathrm{mmol}$ of 6 . Other data for 7: ${ }^{1} \mathrm{H}$ NMR $\left(300 \mathrm{MHz}, \mathrm{C}_{6} \mathrm{D}_{6}, 25{ }^{\circ} \mathrm{C}\right) ; \delta 6.86\left(\mathrm{~s}, 6 \mathrm{H}, \mathrm{C}_{6} \mathrm{H}_{3} \mathrm{Me}_{2}\right.$, $o-\mathrm{H}), 6.60\left(\mathrm{~s}, 3 \mathrm{H}, \mathrm{C}_{6} \mathrm{H}_{3} \mathrm{Me}_{2}, p-\mathrm{H}\right), 2.22\left(\mathrm{~s}, 18 \mathrm{H}, \mathrm{C}_{6} \mathrm{H}_{3} M e_{2}\right), 1.48[\mathrm{~s}, 9 \mathrm{H}$, $\left.\mathrm{C}\left(\mathrm{CD}_{3}\right)_{2} \mathrm{CH}_{3}\right]{ }^{1} \mathrm{H}$ NMR $\left(300 \mathrm{MHz},\left[{ }^{2} \mathrm{H}_{8}\right]\right.$ thf, $\left.25{ }^{\circ} \mathrm{C}\right) ; \delta 6.38(\mathrm{sh} \mathrm{s}, 3 \mathrm{H}$, $\left.\mathrm{C}_{6} \mathrm{H}_{3} \mathrm{Me}_{2}, p-\mathrm{H}\right), 6.25$ (vbr s, $\left.6 \mathrm{H}, \mathrm{C}_{6} H_{3} \mathrm{Me}_{2}, o-\mathrm{H}\right), 2.04$ (sh s, $18 \mathrm{H}$, $\left.\mathrm{C}_{6} \mathrm{H}_{3} M e_{2}\right), \quad 1.31 \quad\left[\mathrm{br} s, 9 \mathrm{H}, \quad \mathrm{C}\left(\mathrm{CD}_{3}\right)_{2} \mathrm{CH}_{3}\right]$. Anal. Calc. for $\mathrm{C}_{37} \mathrm{H}_{36} \mathrm{D}_{18} \mathrm{KN}_{3} \mathrm{Mo}$ : C, 64.04; H, 7.84; N, 6.05. Found: C, 64.71; H, 7.59; N, $5.85 \%$.

$\dagger \dagger$ The reaction to produce 8 was carried out using $0.05 \mathrm{mmol}$ of 7 . Other data for 8: ${ }^{1} \mathrm{H}$ NMR $\left(300 \mathrm{MHz},\left[{ }^{2} \mathrm{H}_{8}\right] \mathrm{thf}, 25{ }^{\circ} \mathrm{C}\right) ; \delta 6.26\left(\mathrm{~s}, 3 \mathrm{H}, \mathrm{C}_{6} \mathrm{H}_{3} \mathrm{Me}_{2}\right.$, $p-\mathrm{H}), 5.71$ (vbr s, $\left.6 \mathrm{H}, \mathrm{C}_{6} \mathrm{H}_{3} \mathrm{Me}_{2}, o-\mathrm{H}\right), 3.58(\mathrm{~m}, 12 \mathrm{H}, \mathrm{crypt}), 3.53(\mathrm{~m}, 12$
$\mathrm{H}$, crypt), $2.53\left(\mathrm{~m}, 12 \mathrm{H}\right.$, crypt), $1.92\left(\mathrm{~s}, 18 \mathrm{H}, \mathrm{C}_{6} \mathrm{H}_{3} M e_{2}\right), 1.50[\mathrm{~s}, 9 \mathrm{H}$, $\left.\mathrm{C}\left(\mathrm{CD}_{3}\right)_{2} \mathrm{CH}_{3}\right]$.

$\ddagger \ddagger$ The reaction to produce 9 was carried out using $0.21 \mathrm{mmol}$ of 7. Other data for 9: ${ }^{1} \mathrm{H}$ NMR $\left(300 \mathrm{MHz},\left[{ }^{2} \mathrm{H}_{8}\right]\right.$ thf, $\left.25{ }^{\circ} \mathrm{C}\right) ; \delta 6.85(\mathrm{~m}, 8 \mathrm{H}$, crown aryl), 6.60 (s, $\left.3 \mathrm{H}, \mathrm{C}_{6} \mathrm{H}_{3} \mathrm{Me}_{2}, p-\mathrm{H}\right), 5.81$ (br s, $\left.6 \mathrm{H}, \mathrm{C}_{6} \mathrm{H}_{3} \mathrm{Me}_{2}, o-\mathrm{H}\right), 3.5-4.1$ $\left(\mathrm{m}, 16 \mathrm{H}\right.$, crown methylene), $2.06\left(\mathrm{~s}, 18 \mathrm{H}, \mathrm{C}_{6} \mathrm{H}_{3} M e_{2}\right), 1.36[\mathrm{~s}, 9 \mathrm{H}$, $\mathrm{C}\left(\mathrm{CD}_{3}\right)_{2}\left(\mathrm{CH}_{3}\right)$ ]. Anal. Calc. for $\mathrm{C}_{65} \mathrm{H}_{52} \mathrm{D}_{18} \mathrm{KMoN}_{3} \mathrm{O}_{10}$ : $\mathrm{C}, 63.44 ; \mathrm{H}, 7.70$; N 3.42. Found: C, 62.83; H, 7.72; N, 3.36\%.

$\S \S$ Details of the X-ray diffraction study of 9: monoclinic, space group $P 2_{1} / n, a=14.094(4), b=31.815(8), c=17.589(7) \AA, \beta=97.60(2)^{\circ}, U$ $=7818(4) \AA^{3}, Z=4$; data/parameter ratio $=10.1, R_{1}=0.0829, w R_{2}$ $=0.1650$, GOF on $F^{2}=1.083$; residuals based on $I>2 \sigma(I)$. CCDC $182 / 596$.

1 C. E. Laplaza, M. J. A. Johnson, J. C. Peters, A. L. Odom, E. Kim, C. C. Cummins, G. N. George and I. J. Pickering, J. Am. Chem. Soc., 1996, 118, 8623.

2 C. E. Laplaza and C. C. Cummins, Science, 1995, 268, 861.

3 M. H. Chisholm, C. E. Hammond, V. J. Johnston, W. E. Streib and J. C. Huffman, J. Am. Chem. Soc., 1992, 114, 7056.

4 K. G. Caulton, R. H. Cayton, M. H. Chisholm, J. C. Huffman, E. B. Lobkovsky and Z. Xue, Organometallics, 1992, 11, 321.

5 R. L. Miller, P. T. Wolczanski and A. L. Rheingold, J. Am. Chem. Soc., 1993, 115, 10422

6 D. R. Neithamer, R. E. LaPointe, R. A. Wheeler, D. S. Richeson, G. D. Van Duyne and P. T. Wolczanski, J. Am. Chem. Soc., 1989, 111 9056.

7 D. Mansuy, J.-P. Lecomte, J.-C. Chottard and J.-F. Bartoli, Inorg. Chem., 1981, 20, 3119.

8 V. L. Goedkin, M. R. Deakin and L. A. Bottomley, J. Chem. Soc., Chem Commun., 1982, 607.

9 S. L. Latesky and J. P. Selegue, J. Am. Chem. Soc., 1987, 109, 4731.

10 M. Etienne, P. S. White and J. L. Templeton, J. Am. Chem. Soc., 1991, 113, 2324.

11 E. M. Carnahan, J. D. Protasiewicz and S. J. Lippard, Acc. Chem. Res., 1993, 26, 90 .

12 G. M. Jamison, A. E. Bruce, P. S. White and J. L. Templeton, J. Am. Chem. Soc., 1991, 113, 5057.

13 M. R. Churchill, A. L. Rheingold and H. J. Wasserman, Inorg. Chem., 1981, 20, 3392.

14 K.-Y. Shih, K. Totland, S. Seidel and R. R. Schrock, J. Am. Chem. Soc., 1994, 116, 12103.

15 J. Manna, R. J. Kuk, R. F. Dallinger and M. D. Hopkins, J. Am. Chem. Soc., 1994, 116, 9793.

16 P. R. Sharp, S. J. Holmes, R. R. Schrock, M. R. Churchill and H. J. Wasserman, J. Am. Chem. Soc., 1981, 103, 965.

17 M. H. Chisholm, K. Folting, D. M. Hoffman and J. C. Huffman, J. Am. Chem. Soc., 1984, 106, 6794.

18 R. R. Schlosser and J. Hartmann, Angew. Chem., Int. Ed. Engl., 1973, 12, 508.

19 C. P. Casey, M. W. Meszaros, P. J. Fagan, R. K. Bly, S. R. Marder and E. A. Austin, J. Am. Chem. Soc., 1986, 108, 4043; see this reference and ref. 20 for a ${ }^{13} \mathrm{C}$ NMR shift of $c a .500 \mathrm{ppm}$.

20 C. P. Casey, M. A. Gohdes and M. W. Meszaros, Organometallics, 1986, 5, 196

21 E. Weiss, Angew. Chem., Int. Ed. Engl., 1993, 32, 1501.

22 J. C. Peters, A. L. Odom and C. C. Cummins, unpublished work.

23 M. G. Fickes, A. L. Odom and C. C. Cummins, Chem. Commun., 1997, this issue; see this paper for structural data pertaining to $[\mathrm{Na}\{\mathrm{NNb}[\mathrm{N}$ (R)Ar $\left.\left.]_{3}\right\}\right]_{2}$, which is analogous structurally to $\left[\mathrm{K}\left\{\mathrm{CMo}[\mathrm{N}(\mathrm{R}) \mathrm{Ar}]_{3}\right\}\right]_{2}$.

24 L. F. Lindoy, The Chemistry of Macrocyclic Ligand Complexes, Cambridge University Press, Cambridge, 1989.

25 Comprehensive Supramolecular Chemistry, ed. G. W. Gokel, Pergamon, New York, 1996.

26 W. A. Nugent and J. M. Mayer, Metal-Ligand Multiple Bonds, Wiley, New York, 1988.

27 C. A. Hunter and J. K. M. Sanders, J. Am. Chem. Soc., 1990, 112, 5525.

28 C. E. Laplaza, W. M. Davis and C. C. Cummins, Angew. Chem., Int. Ed. Engl., 1995, 34, 2042.

Received in Bloomington, IN, USA, 17th June 1997; 7/04251E 